\title{
Editorials
}

\section{Homocysteine and cardiovascular disease}

There are many risk associations with premature cardiovascular disease, some of which relate to lifestyle and are potentially modifiable. These now include raised plasma concentrations of homocysteine (hyperhomocysteinaemia; $\mathrm{HHC}$ ) and its derivatives, and Still and $\mathrm{McDowell}^{1}$ have summarised present information on and recommend guidelines on the pre-analytical, methodological, and standardisation concerns to be addressed if the association is to be taken further. The whole naturopathic movement can be seen as very Californian, with HHC now displacing selenium, vitamin $\mathrm{E}$, and antioxidants as the in thing. But associations with HHC have been reported consistently in a wide spectrum of observational and prospective studies, with about $10 \%$ of the population risk of coronary heart disease attributable to $\mathrm{HHC}^{2}$ The association was first revealed through the pathology of homozygous homocystinuria, with intravascular thrombosis. Earlier suggestions from small trials that $\mathrm{HHC}$ had a particular association with extracoronary vascular disease have not been confirmed by larger studies; all arterial and cerebrovascular events seem to be involved and with greater impact in the presence of other cardiovascular risk associations, particularly diabetes. ${ }^{3}$ The mechanisms of cardiovascular damage through HHC are not exactly defined but seem to include endothelial damage and platelet aggregation, associated with the production of modified forms of low density lipoprotein, and stimulation of arterial smooth muscle cell growth. The breakdown of fibrillar structures such as the lens zonule were sufficiently characteristic to lead to a diagnosis postmortem. ${ }^{4}$ The evidence that homocysteine itself is the toxic agent is persuasive, as supplements of pyridoxine, cobalamin, and particularly folate can promote the metabolic pathways of remethylation or conversion to cystine and show clear benefits on plasma biochemistry and clinical course in genetic homocystinuria. Vitamin responsiveness to less specific forms of HHC are now under intense investigation. The evidence for gene-environment interaction here is also persuasive. First, heterozygotes for classic homocystinuria have variable but generally acceptable concentrations of homocysteine in plasma and are not at obviously excess risk of cardiovascular events. ${ }^{5}$ Second, a common variant reduced activity form of the remethylation enzyme, methyl tetrahydrofolate reductase (MTHFR), has a variable and with some ethnic groups no association with either HHC or premature cardiovascular events. ${ }^{6}$ Associations may depend on the level of folate also present, and HHC could indeed be a marker for deficiency of $\mathrm{B}$ vitamins and particularly folate which could drive the vascular pathology. ${ }^{7}$ There are difficulties in defining reference ranges for both homocysteine and folate, as well as concerns that measurements on single samples may not be adequate to review relations with outcome years later. Population data for homocysteine show a distribution skewed to the right, with a median slightly increasing with age and higher in males, and with an association with cardiovascular events increasing above about $13 \mu \mathrm{mol} / \mathrm{litre}$. Folate supplementation can reduce HHC by at least $25 \%$, with a further $7 \%$ through additional supplementation with vitamin B-12. ${ }^{7}$ Pyridoxine effects are only reported for some patients with classic homocystinuria. Unexpectedly, folate supplementation in general reduces homocysteine at all starting levels and with optimal effects at a daily intake of $>400 \mu \mathrm{g} /$ day, through which some $88 \%$ of the US population would be defined as folate deficient. ${ }^{78}$ The habit of taking vitamin and other health food supplements, and of fortifying flour, is much more prevalent in North America than in Europe, and wide differences in vitamin status may explain why a range of observational studies has produced rather mixed results. It is not certain whether folate or another component present in folate-rich food is the prime agent, and also whether HHC acting directly or as a marker shows a threshold effect. But it is proposed that reducing cardiovascular risk through vitamin supplementation could be a great deal easier to implement, more acceptable, and cheaper than interventions to lower cholesterol, and the epidemiology is consistent with a reduction in vascular events of up to $40 \%$. $^{2}$

However, this is guilt by association-extrapolation based medicine analogous to the fact that more than $90 \%$ of victims in car accidents are wearing shoes. Still and $\mathrm{McDowe}^{1}{ }^{1}$ emphasise that specialist cardiovascular and perhaps renal laboratories may take an interest but that routine homocysteine assays are not yet with us because clinical significance is not established and there are methodological difficulties. Appropriate randomised intervention trials are in hand.

A F WINDER

Academic Department of Chemical Pathology and Human Metabolism Royal Free NHS Hospital Trust and School of Medicine (University of London), London NW3 2QG, UK; email: tony_w@rfhsm.ac.uk

1 Still RA, McDowell IFW. Clinical implications of plasma homocysteine measurement in cardiovascular disease. ACP Broadsheet 152. F Clin Pathol 1998;51:183-8.

2 Boushey CJ, Beresford SA, Omen GS, et al. A quantitative assessment of plasma homocysteine as a risk factor for vascular disease. Probable benefits of increasing folic acid intakes. $\mathscr{F} A M A 1995 ; 274: 1040-57$.

3 Hoogeveen EK, Kostense PJ, Beks PJ, et al. Hyperhomocysteinemia is associated with an increased risk of cardiovascular disease, especially in non-insulin-dependent diabetes mellitus. A population-based study. Art Thromb Vasc Biol 1998;18:133-8.

4 Henkind P, Ashton N. Ocular pathology in homocystinuria. Trans Ophthalmic Soc UK 1965;85:21-38.

5 Mudd SH, Havlik R, Levy HC, et al. A study of cardiovascular risk in het-

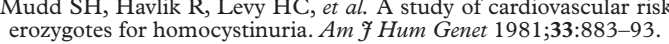

6 Ou TA, Yamakawa-Kobayashi K, Arinami T, et al. Methylenetetrahydrofolate reductase and apolipoprotein E polymorphisms are independent risk factors for coronary heart disease in Japanese: a case-control study. Atherosclerosis 1998;137:23-8.

7 Clarke R, for the Homocysteine Lowering Triallists Collaboration. Lowering blood homocysteine with folic acid based supplements: meta-analysis of randomised trials. BMf 1998;316:894-8.

8 den Heijer M, Brouwer IA, Bos GMJ, et al. Vitamin blood supplementation reduces blood homocysteine levels. A controlled trial in patients with venous thrombosis and healthy volunteers. Art Thromb Vasc Biol 1998;18:356-61. 


\section{Telepathology: assessment of the implications and applications of telepathology for practical diagnostic pathology}

The concept of using video microscopy to provide diagnostic services to remote locations was first described in the USA in 1968, when monochrome images were transmitted in real time using a dedicated point to point microwave link. In little more than a decade telepathology has developed from the prototype commercial system first described in 1986 to today's multimedia computers which can be purchased "off the shelf" at very low prices and can be used as the basis of telepathology systems. ${ }^{1}$

There are technological problems which may prevent the acceptance of telepathology by pathologists and thus hinder its application within the routine environment. Some pathologists believe that telepathology is too expensive and that it does not have a useful role in routine pathology. Others have doubted if computer monitors can be used for making a diagnosis, although results have shown no significant difference between a pathologist's performance using a microscope and using a video image. Many of the concerns expressed about the use of telepathology are not derived from knowledge. There is a need to involve as many pathologists as possible in the use of these systems, particularly pathologists working in routine service roles.

Many pathologists believe that fast access to expert opinion is the key to reducing the numbers of diagnostic errors, which have been estimated at up to $5 \% .^{2}$ Although most of these are not necessarily critical to the patient, some could be.

In 1992 the 1st European Symposium on Telepathology was held in Heidelberg. Telepathology was used in Scandinavia for remote diagnosis of frozen sections and elsewhere in Europe, Japan, and the USA for histology and cytology. ${ }^{3}$

Since 1995 there has been worldwide interest in the research, development, and application of telepathology. Public awareness of telepathology was raised in October 1996 by an article in The Guardian newspaper, entitled "Second opinions from space," which described the use of telepathology at the Norwegian Radium Hospital. ${ }^{2}$ A European Union (EU) research project (EUROPATH) involving many of the member states began in 1996 and in January 1997 a whole edition of a major pathology journal was devoted to telepathology. ${ }^{4}$

Currently research projects are investigating the use of telepathology for the implementation of multimedia databases, remote consultation, remotely driven microscopy, remote diagnosis, referrals, consensus teleconferences, quality assurance, and teaching.

At present the routine use of telepathology systems in parts of Europe appears to be directed at remote frozen section diagnosis. In the United Kingdom, however, frozen sections make up only a small percentage of laboratory cases and the rate is falling. If this trend spreads then frozen section diagnosis by telepathology may not be the most important application. Other applications - currently the subject of research and development-are remote diagnosis, consensus diagnosis, teaching, and quality control.

The purchase cost of a fully automated remote telepathology system remains high. Data transmission costs are very high for those without fast internet access. These high costs and potentially infrequent use are the major obstacles to remote control remote diagnosis. The use of remote instruction for the control of the microscope is a cheaper alternative. This method, however, is not free of problems.

Consensus diagnosis, considered by some to be ideally suited to telepathology, is not at present available to those most likely to need it. The large bandwidth required is either not readily available, too expensive, or both! This is unfortunate since the telepathology systems currently available are capable of providing image displays that are ideal for consensus diagnosis.

Substantially cheaper communication methods can be used where images are sent before consultation. Such applications include teaching, quality assurance, accessing databases, and "earliest convenience" diagnosis.

All communication costs, with the exception of the standard telephone, are very expensive and represent a major obstacle to the routine application of telepathology.

The most commonly used telecommunications are the public service telephone network (PSTN), using a modem, and the integrated services digital network (ISDN). ${ }^{5} \mathrm{~A}$ search of published reports indicates that ISDN is probably the most widely used protocol, although in the United Kingdom it is expensive to install and use. Data transfer speed using the PSTN has recently improved and is potentially equivalent to one ISDN channel. The development of image compression, which has been claimed to reduce the digital image size by about 50 times without eroding the picture quality, has renewed interest in this inexpensive form of data transmission.

Network communications, especially the internet, provide an inexpensive method of communication, even over long distances. The bandwidth of the internet is increasing and fast data transmission is possible. Local area networks, which can be very fast and enable real time communications, exist in universities and hospitals. The cost to individual departments is negligible, but a major disadvantage is that academic networks and clinical networks may not be allowed to link with each other owing to security considerations, which may also apply to the internet. Internet access for NHS hospitals in the United Kingdom is still a major problem and the variable speed of data transmission caused by the continuing use of old technology is an additional problem. Nevertheless many telepathology applications are now suited to this protocol. Satellite links offer the highest data transmission speeds, but their very high operational costs prevent them from being in common use.

Some pathologists have expressed an opinion that real time image displays are not only preferable but necessary for remote applications. Real time applications are only possible when larger bandwidths are available. The costs of purchase and running of these systems are largely unsupportable by ordinary laboratories. This may change in the near future. Near real time-the exchange of images in less than five seconds-may permit on-line discussions of cases between two pathologists. It has been argued that only real time selection of images can fulfil the legal requirements. Pathologists willing to give a second opinion on static images not selected by them should save these images as a legal record of what they saw. Equally, with real time selection, the images selected should be stored. The medicolegal aspects of who is responsible for the diagnosis in a referral case are unclear. Ultimately the responsibility for issuing a report depends on the referring pathologist, 
taking into account the expert opinion. The argument that the referring pathologist has not included the diagnostic image could equally apply to recut slides sent for opinion, and is not confined to telepathology images. This appears to be a largely theoretical problem which has not yet been resolved but which should not be allowed to hinder development.

Further research and development is therefore needed, but a lack of funding is the major problem, making the implementation of telepathology difficult or impossible for many laboratories. Such implementation is vital to assess the unknown factors which may reduce the usefulness of telepathology. Only by using telepathology can specifications for pathological applications be determined. Moreover, such specifications must be validated and assessed in order to determine their usefulness and true costs. A call for industry standards, to enable systems from different manufacturers to communicate with each other, has been included in recent research projects. It appears that the manufacturers themselves are unlikely to achieve the compatibility needed. If this is to be done by a third party some investment will have to be made.

Indications are that seeking a specialist opinion on cases of difficulty will be quicker and easier in the future. The development of reference banks of quality assured images and international cancer databases will be used to improve diagnostic accuracy and continuing postgraduate education (CME). Telepathology will also enable the interactive discussion of referral cases, leading to more secure pathological diagnosis in difficult cases. Such a wide user base would enable speedier dissemination of information. Pathologists seeking expert opinion would be able to derive the same benefits from a consultation as those using multiheaded microscopes.

The EUROpean Pathology Assisted by Telematics for Health (EUROPATH project No HC - 1038 of DGXIII) is supported by the European Union (project coordinator Professor G Brugal, Grenoble).

C SOWTER

C A WELLS

Department of Histopathology, St Bartholomew's Hospital,

Royal Hospitals Trust, London EC1A 7BE, UK

1 C Sowter, P Domizio, CA Wells, et al. Quality assurance using telepathology and standard telecommunications: a viable alternative to the Post Office? $\mathcal{F}$ Pathol 1997;181(suppl):27.

2 Marks P. Second opinions from space. The Guardian 1996; October 16.

3 Proceedings of the 1st European Symposium on Telepathology. Zentralbl Pathol 1992;138,381-436.

4 Telepathology symposium. Hum Pathol 1997;28:1-118.

5 British Telecommunications Information. ISDN: many applications; a single tool. London: British Telecommunications, 1993. 